\title{
INDICADORES CLÍNICO-EPIDEMIOLÓGICOS MATERNO-FETALES DE ATONÍA UTERINA EN PUÉRPERAS POST CESÁREA PRIMARIA EN UNA CLÍNICA PRIVADA DE AGOSTO 2017 - AGOSTO 2018
}

\author{
MATERNO-FETAL CLINICAL-EPIDEMIOLOGICAL INDICATORS OF UTERINE ATONY IN PUERARPAS POST \\ PRIMARY CESÁREA IN A PRIVATE CLINIC OF AUGUST 2017 - AUGUST 2018
}

María Y. Ponce de León-Galarza'

\begin{abstract}
RESUMEN
Objetivo: Determinar la asociación entre indicadores clínico - epidemiológicos materno - fetales y la presencia de atonía uterina en puérperas post cesárea primaria en la clínica privada durante el período de Agosto 2017 - Agosto 2018. Métodos: Se realizó un estudio observacional, analítico, retrospectivo y cuantitativo, tipo casos y controles. La variable dependiente fue la atonía uterina, y las variables independientes fueron edad materna, gestación múltiple, polihidramnios, gestación, paridad, anemia materna, enfermedades concomitantes, peso fetal, presentación fetal e indicación de cesárea. La población estuvo conformada por 106 casos y 212 controles (ratio 2:1). Para la estadística inferencial se utilizó el análisis bivariado para hallar los Odds Ratio, intervalos de confianza al 95\%. Se consideró a los valores $\mathrm{p}<0,05$ como estadísticamente significativos. Resultados: De los 318 pacientes del estudio, la media de la edad fue de 30,87 $\pm 0,298$ años, de los controles fue de $28 \pm 0,423$ años mientras que de los casos $33 \pm 0,168$ años. Las variables asociadas en el análisis bivariado a atonía uterina fueron la edad mayor de 30 años, gestación múltiple, polihidramnios, bajo peso al nacer y macrosomía. Mientras que en análisis multivariado fueron polihidramnios (OR: 5,973 IC95\%: 2,443-14,603) y macrosomía (OR: 6,280 IC95\%: 2,307-17,095). Conclusión: Se concluye que, se encontró asociación de atonía uterina con los indicadores de polihidramnios y macrosomía fetal
\end{abstract}

Palabras clave: Inercia uterina; Hemorragia uterina; Polihidramnios, Macrosomía fetal. (fuente: DeCS BIREME)

\begin{abstract}
Objective: To determine the association between maternal - fetal clinical - epidemiological indicators and the presence of uterine atony in post - caesarean primary puerperae at the Good Hope clinic during the period of August 2017 - August 2018. Methods: An observational, analytical, retrospective and quantitative study, type of cases and controls was carried out. The dependent variable was uterine atony, and the independent variables were maternal age, multiple gestation, polyhydramnios, pregnancy, parity, maternal anemia, concomitant diseases, fetal weight, fetal presentation and indication of cesarean section. The population consisted of 106 cases and 212 controls (ratio 2: 1). For the inferential statistics, the bivariate analysis was used to find the Odds Ratio, 95\% confidence intervals. The values $p<0.05$ were considered statistically significant. Results: Of the 318 patients in the study, the mean age was $30.87 \pm 0.298$ years. Associated variables to uterine atony in the bivariate analysis were age over 30 years, multiple gestation, polyhydramnios, low birth weight and macrosomia. In multivariate analysis were polyhydramnios (ORa: 5,973,95\% Cl:2,443-14,603) and macrosomia (ORa:6.280,95\% Cl:2.307-17.095). Conclusion: It is concluded that, association of uterine atony with polyhydramnios indicators and fetal macrosomia was found.
\end{abstract}

Key words: Uterine Inertia, Uterine Hemorrhage, Polyhydramnios, Fetal Macrosomia. (source: MeSH NLM) 


\section{INTRODUCCIÓN}

Desde principios del siglo XX, la mayor amenaza para la seguridad de las mujeres en edad fértil ha sido la hemorragia posparto (HPP), que es la principal causa de morbilidad y mortalidad materna en los países de ingresos bajos y medios (LMIC) y una de las principales causas de morbilidad en países de altos ingresos. En todo el mundo, la HPP severa (definida como pérdida de $1000 \mathrm{ml}$ de sangre) afecta a 1,6 millones de mujeres y representa de 130000 a 140000 muertes maternas cada año, lo que significa que hay aproximadamente 380 muertes cada 24 horas o aproximadamente 1 muerte materna por cada día del año'.

Según la Organización Mundial de la Salud (OMS) ocurren alrededor de 529000 muertes maternas al año, ocupando un lugar prioritario en planteamientos políticos nacionales e internacionales'. Sirve además, como reflejo de la desigualdad social, inequidad de género y evidencia la falta de capacidad de respuesta de un sistema de Salud.

En el documento presentado por la Dirección General de Epidemiología (DGE) del Ministerio de Salud de Perú se describe la razón de mortalidad materna promedio durante el 2002-2011, la cual fue de 111.92, que si bien ha presentado tendencia a la disminución en la última década, las cifras continúan siendo una de las más altas de América Latina en donde cada año se describen alrededor de 34000 muertes maternas, de las cuales la mayoría ocurre en Sudamérica. En el año 2015 el Perú ocupó el sexto lugar dentro de los países con tasas elevadas de mortalidad materna, después de Guyana, Bolivia, Surinam, Paraguay y Venezuela ${ }^{12}$.

Es importante destacar que en la última década, la mortalidad materna en Perú ha disminuido en un $66 \%$ a 67 muertes por cada 100000 nacidos vivos ${ }^{4}$ y el país está en camino de cumplir el propósito de los objetivos del milenio de una reducción del $75 \%$ para $2015^{2}$. Durante este mismo período, la mortalidad materna en realidad ha aumentado en algunos países de ingresos altos $^{5,6}$. Sin embargo, aunque Perú ha logrado avances impresionantes, la HPP sigue siendo la causa del $40 \%$ de las muertes maternas, y las disparidades entre las áreas indígenas urbanas y rurales continúan.

Son diversas las causas de hemorragia post parto, la lista la encabeza la atonía uterina y en segundo lugar las enfermedades hipertensivas del embarazo (preeclampsia y eclampsia) $^{9}$, es necesario conocer los principales determinantes de la atonía uterina en nuestra población, para poder actuar de forma oportuna cuando este se presente y aplicar todos los protocolos necesarios para prevenirla.

El objetivo principal del presente estudio fue determinar la asociación entre indicadores clínico epidemiológicos materno - fetales y la presencia de atonía uterina en puérperas post cesárea primaria en una Clínica Privada de Lima-Perú durante el período de Agosto 2017 - Agosto 2018.

\section{MÉTODOS}

Estudio observacional, analítico, retrospectivo y cuantitativo de tipo casos y controles. La población estuvo conformada por 318 pacientes puérperas post cesárea primaria del servicio de Ginecología de la clínica Good Hope, durante los meses de Agosto del 2017 a Agosto del 2018.

Se trabajó con una relación caso-control de 1:2, con 106 casos y 212 controles, mujeres con diagnóstico de atonía uterina descrito en el reporte intraoperatorio. Se excluyeron a aquellas pacientes con antecedente de cesárea previa o cualquier otra cirugía uterina y a aquellas con historias clínicas con datos incompletos maternos $\mathrm{y} / \mathrm{o}$ fetales.

Se utilizó la documentación como técnica de recolección de datos, registrados en historias clínicas mediante el programa Quick View y CIE10. Los datos recopilados fueron ingresados a una base de datos en SPSS versión 23.

Se utilizó el análisis bivariado para hallar los Odd Ratio, con sus respectivos intervalos de confianza al 95\% y una significancia p $(<0.05)$ mediante la prueba Chi cuadrado. Finalmente se realizó análisis multivariado para obtener OR ajustados.

\section{RESULTADOS}

Se recolectó información de 318 historias clínicas y reportes quirúrgicos; donde se encontró que la media de edad total fue de $30,87 \pm 0,298$ años, de los controles fue de $28 \pm 0,423$ años mientras que, de los casos fue de $33 \pm 0,168$ años. La principal indicación de cesárea fue la desproporción céfalo-pélvica con 33,3\% y el sufrimiento fetal agudo con 7,9\%. Con respecto a las enfermedades concomitantes durante la gestación se encontró que la más frecuente fue la miomatosis uterina con $7,9 \%$. La mayoría de pacientes, el $60.7 \%$, no tuvo presencia de enfermedades concomitantes. Tabla 1. 
Tabla 1. Análisis bivariado de los factores asociados a atonía uterina.

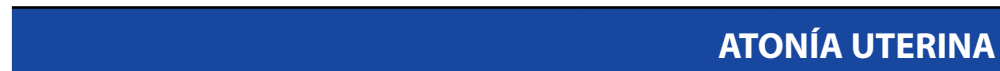

S

\section{OR (IC 95\%)}

VALOR P

Edad

Mayor de 30 años

$\begin{array}{lll}66 & 99 & 165 \\ 40 & 113 & 153\end{array}$

$1,88(1,17-3,03)$

0,009

30 años o menos

NO TOTAL

165

53

Gestación múltiple

Si

$$
5
$$

No

101

1

6

$10,45(1,20-90,58)$

0,017

Polihidramnios

$\mathrm{Si}$

19

8

27

$\begin{array}{lll}87 & 204 & 291\end{array}$

$5,57(2,35-13,20)$

0,001

No

204

Paridad

Multípara

9

29

38

Primípara

97

183

280

$0,58(0,26-1,28)$

0,203

Enfermedad concomitante

$\mathrm{Si}$

43

82

125

No

63

130

193

$1,08(0,67-1,74)$

0,808

Bajo peso al nacer

$\mathrm{Si}$

No

$7 \quad 3$

3

10

99

209

308

$4,92(1,25-19,45)$

0,018

Macrosomía fetal

Si

$15 \quad 6 \quad 21$

No

91

206

297

$5,66(2,13-15,05)$

0,001

Anemia

Si

$18 \quad 24$

No

88

188

42

$1,60(0,83-3,10)$

0,164

Presentación cefálica

$\mathrm{Si}$

73

161

234

No

33

51

84

$0,70(0,42-1,17)$

0,180

En lo que respecta a la gestación múltiple, se encontró que el 4,72\% (5) de las puérperas que tenían atonía uterina presentaban gestación múltiple, siendo esta relación estadísticamente significativa $(p=0.017)$.

Asimismo, se encontró que las puérperas con gestación múltiple, tienen 10,45 veces de tener atonía uterina que las puérperas sin gestación múltiple, tabla 2. 
Tabla 2. Relación entre gestación múltiple y la presencia de atonía uterina.

\begin{tabular}{|c|c|c|c|c|c|}
\hline \multirow[b]{2}{*}{$\begin{array}{l}\text { GESTACIÓN } \\
\text { MÚLTIPLE }\end{array}$} & \multicolumn{3}{|c|}{ ATONÍA UTERINA } & \multirow[t]{2}{*}{ OR (IC 95\%) } & \multirow[t]{2}{*}{ VALOR F } \\
\hline & SI & No & TOTAL & & \\
\hline $\mathrm{Si}$ & $5(4.72 \%)$ & $1(0.47 \%)$ & $6(1.88 \%)$ & & \\
\hline No & 101 (95.28\%) & 211 (99.53\%) & 312 (98.12\%) & $\begin{array}{c}10,45 \\
(1,20-90,58)\end{array}$ & 0,017 \\
\hline TOTAL & 106 & 212 & 318 & & \\
\hline
\end{tabular}

Además, con respecto al polihidramnios, se encontró uterina presentaban polihidramnios; siendo esta relación que el $17,92 \%$ (19) de las puérperas que tenían atonía estadísticamente significativa ( $p=0.001)$, figura 1.

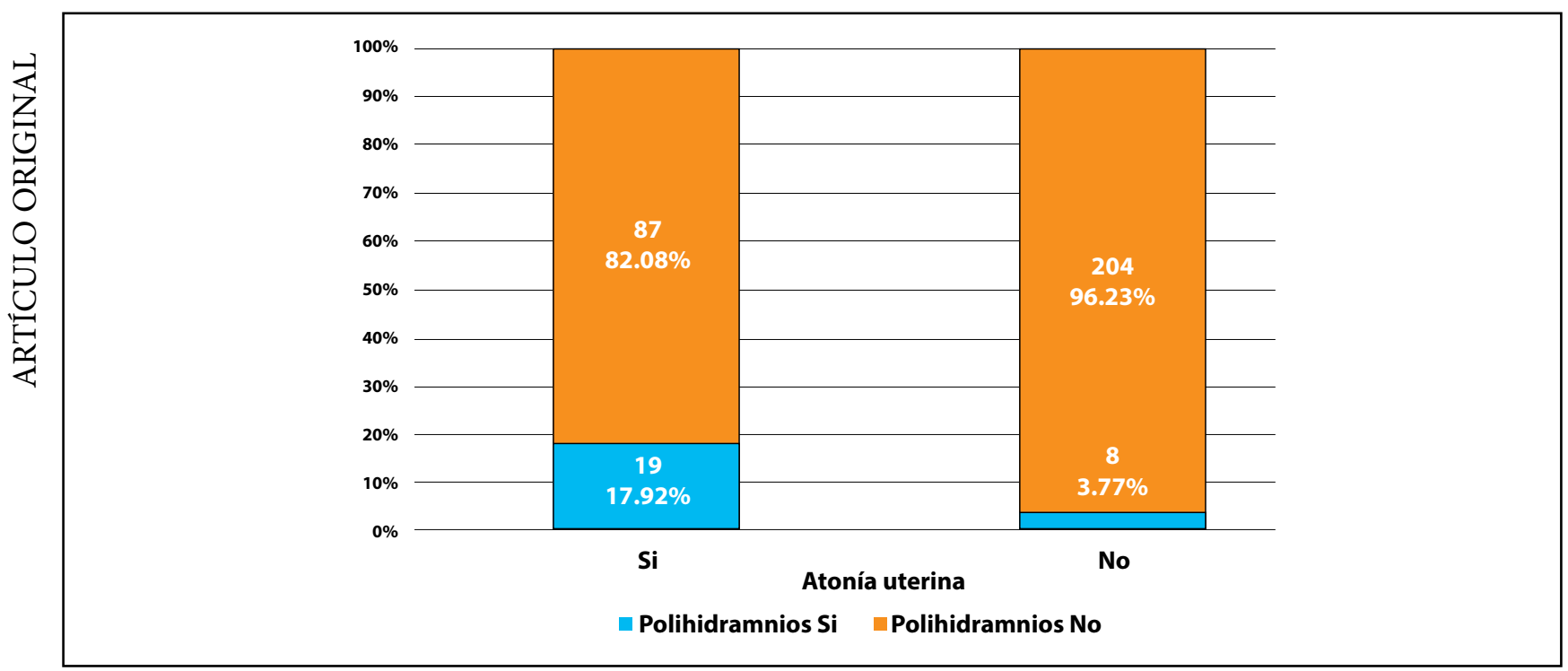

Gráfico 1. Relación entre polihidramnios y la presencia de atonía uterina.

Con respecto al bajo peso al nacer, solo el 3.14\% (10) del total de puérperas (318) tuvieron hijos con bajo peso al nacer. En el análisis se encontró que el 6,6 \% (7) de las puérperas que presentaban atonía uterina tenían hijos con bajo peso al nacer, siendo esta relación estadísticamente significativa $(p=0.018)$. Finalmente, en el análisis entre macrosomía, se encontró que el $14,15 \%$ (15) de las puérperas que tenían atonía uterina tenían hijos con macrosomía fetal.

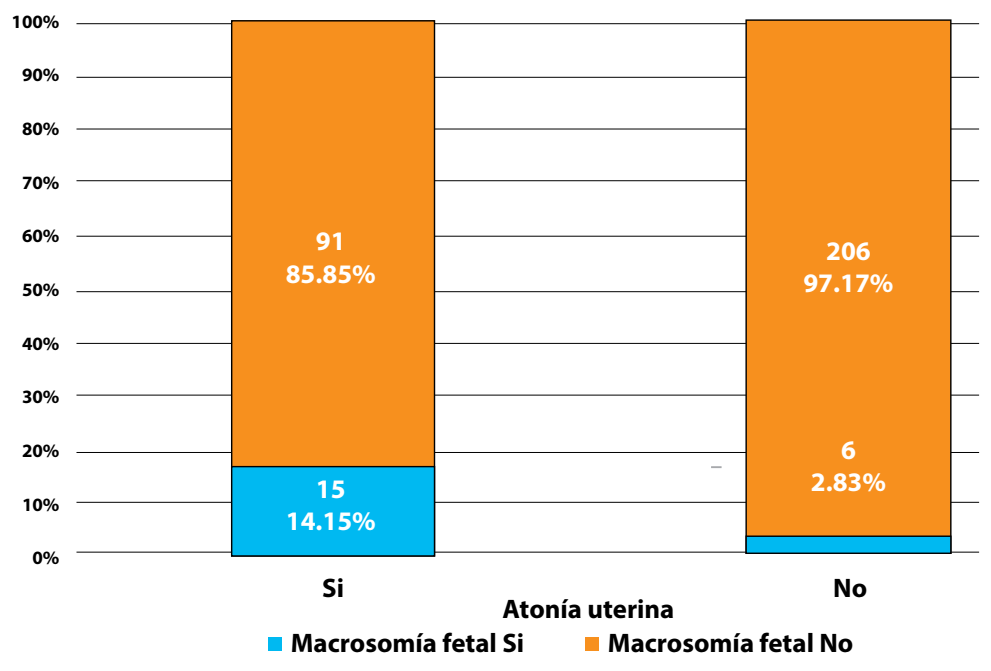

Gráfico 2. Relación entre macrosomía y la presencia de atonía uterina. 
Tabla 3. Análisis multivariado de los factores asociados a atonía uterina.

\begin{tabular}{lcccc} 
& OR AJUSTADO & \multicolumn{2}{c}{ IC 95 \% } & VALOR P \\
& & LíMITE INFERIOR & LímITE SUPERIOR &, 052 \\
\hline Edad mayor de 30 años & 1,672 &, 996 & 2,806 &, 104 \\
Gestación múltiple & 6,522 &, 679 & 62,629 &, 000 \\
Polihidramnios & 5,973 & 2,443 & 14,603 &, 074 \\
Bajo peso al nacer & 3,911 &, 876 & 17,465 &, 000 \\
\hline Macrosomía & 6,280 & 2,307 & 17,095 & \\
\hline
\end{tabular}

\section{DISCUSIÓN}

La muerte materna es uno de los más importantes problemas de salud pública, la atonía uterina es una de las principales causas de muerte materna y es evitable. En el presente estudio, se encontró que la edad mayor de 30 años es un factor de riesgo para atonía uterina; con un OR de 1,88. Sin embargo, esta relación no se mantuvo al momento de hacer el análisis multivariado, probablemente por falta de potencia estadística. Este resultado es similar a los encontrados en otros estudios, como el trabajo de Cavazos et al. en Estados Unidos donde se encontró que las mujeres entre 15 y 19 años tenían más riesgo de hemorragias posparto, mientras que las mayores de 35 años no presentaban más riesgo de hemorragia posparto ${ }^{23}$. Asimismo, otro estudio realizado por Augusto M. et al. en el Hospital Almenara en Lima, Perú, encontró que los factores de riesgo para atonía uterina fue, entre otros, tener una edad mayor a 30 años con un OR de 3,64 ${ }^{16}$. Por último, un estudio también realizado en Perú, encontró que en una población de Villa El Salvador hubo mayor prevalencia de atonía uterina en mujeres de entre 19 y 25 años, mientras que en las mujeres mayores de 35 años no hubo diferencia estadísticamente significativa con respecto a la atonía uterina ${ }^{15}$.

Asimismo, hubo una relación estadísticamente significativa con la gestación múltiple y la atonía uterina, sin embargo, esta relación no mantuvo su significancia estadística en el análisis multivariado. Un estudio de casos y controles realizado por Butwick $A$. et al. en Estados Unidos el año 2017 encontró también que los embarazos múltiples fueron un factor de riesgo para hemorragia posparto (con un OR ajustado de 8), así también fue factor de riesgo para cesárea intraparto (con un OR ajustado de 3,2) ${ }^{14}$. Resultado similar fue encontrado por Butwick $A$. et al. en otro estudio realizado en Estados Unidos que se publicó en el 2014, donde la gestación múltiple tuvo un OR ajustado de 1,59 para atonía uterina en pacientes que se sometieron a cesárea ${ }^{20}$. Así también, otro estudio realizado en Perú, en el Hospital Guillermo Almenara Irigoyen en la ciudad de Lima, Perú; encontró que la gestación múltiple tuvo un $\mathrm{OR}$ de 2,15 para atonía uterina, el cual es un OR inferior al encontrado en nuestro estudio; sin embargo, no hay datos acerca de un OR ajustado en este estudio con respecto a esta variable $^{16}$.

En lo que respecta a polihidramnios, se encontró una asociación significativa en el análisis bivariado, con un OR crudo de 5,57; significancia que se mantuvo en al análisis multivariado con un OR ajustado de 5,97. Esta diferencia puede deberse a que las arterias y venas uterinas que tienen su recorrido entre las células musculares del miometrio tienen como mecanismo de hemostasia la contracción de estas fibras que originan su oclusión con el consecuente parado del sangrado; sin embargo, la condición del polihidramnios causa una distención uterina que altera la correcta contracción de sus fibras musculares, causando lo que conocemos como atonía uterina, lo cual hace que el sangrado uterino no pueda ser controlado causando una hemorragia posparto que puede llegar a ser mortal. No existen muchos estudios que hayan explorado esta relación. Uno realizado en el Sultanato de Oman no encontró relación entre la hemorragia postparto y la presencia de polihidramnios, esto probablemente porque se realizó un estudio con una prevalencia muy baja de hemorragia posparto, de solo un $2,7 \%{ }^{22}$. Sin embargo, otro estudio realizado en Estados Unidos por Wetta et al. encontró que si hay relación entre el polihidramnios y la atonía uterina y la hemorragia ${ }^{24}$

En lo que respecta al peso, se halló que el bajo peso al nacer estuvo asociado a atonía uterina en este estudio; sin embargo esta relación no se mantuvo en la estadística multivariada. Un estudio realizado por Nair et al. en la India encontró que los recién nacidos 
con bajo peso tenían un OR ajustado de 8,72 frente a los que tenían peso adecuado ${ }^{17}$.

Con relación al peso, se encontró que la macrosomía estuvo relacionada con la atonía uterina. Esto concuerda con lo reportado por Said et al. en un estudio en Tanzania, donde encontró que una de las complicaciones más frecuentes de los bebes macrosómicos era la hemorragia posparto $(17,5 \%)$ y que esta era 5 veces más frecuente en macrosómicos que en aquellos con peso normal ${ }^{25}$. Otro estudio, esta vez longitudinal de cohortes a un grupo de bebes macrosómicos y a otros no macrosómicos, encontró que en Irán, una de las asociaciones más significativas con respecto a la macrosomía fetal fue la mayor incidencia de atonía uterina ${ }^{24}$. De la misma forma, el estudio desarrollado por Machado et al. en las puérperas del hospital Uldarico Rocca Fernandez de Villa El Salvador encontró que uno de los factores de riesgo para atonía uterina estadísticamente significativos fue la macrosomía fetal ${ }^{15}$.

Correspondencia: María Ysabel Ponce de León

Dirección: 1328 Juan Bielovucic, Lince, Lima - Perú

Teléfono: +51998837377

Correo:mariaysabel_2693@hotmail.com

\section{CONCLUSIÓN}

Se concluye que, la edad materna, gestación múltiple, y el polihidramnios son factores de riesgo para el desarrollo de atonía uterina en pacientes puérperas post cesárea segmentaria primaria. Además, del bajo peso al nacer y la macrosomía fetal también se encuentran asociados como factores de riesgo de atonía uterina.

Contribuciones de autoría: El autor participó en la génesis de la idea, diseño de proyecto, recolección e interpretación de datos, análisis de resultados, preparación del manuscrito, presente trabajo de investigación.

Financiamiento: Autofinanciado.

Conflicto de interés: El autor declara no tener conflicto de interés.

Recibido: 07 octubre de 2018

Aprobado: 22 de enero 2019

\section{REFERENCIAS BIBLIOGRÁFICAS}

1. WHO. Organización mundial de la Salud. Evolución de la mortalidad materna: 1990-2015 Estimaciones de la OMS, el UNICEF, el UNFPA, el Grupo del Banco Mundial y la División de Población de las Naciones Unidas. 2015;2.

2. Maguiña G. La mortalidad materna en el Perú, 2002-2011 [Internet] Lima; 2013 [cited 2018 Aug 28]. Available from: www.dge.gob.pe

3. Távara Orozco L. Tendencia de la mortalidad materna en el Perú: desafíos pendientes . Vol. 59, Revista Peruana de Ginecología y Obstetricia . scielo ; 2013. p. 153-6.

4. Say L, Chou D, Gemmill A, Tunçalp Ö, Moller AB, Daniels J, et al. Globa causes of maternal death: A WHO systematic analysis. Lancet Glob Heal. $2014 ; 2(6)$.

5. CallaghanWM, Kuklina EV., Berg CJ.Trends in postpartum hemorrhage: United States, 1994-2006. Am J Obstet Gynecol [Internet]. 2010 Apr [cited 2018 Aug 28];202(4):353.e1-353.e6. Available from: https://www. sciencedirect.com/science/article/pii/S0002937810000220

6. von Schmidt auf Altenstadt JF, Hukkelhoven CWPM, van Roosmalen J, Bloemenkamp KWM. Pre-eclampsia increases the risk of postpartum haemorrhage: a nationwide cohort study in the Netherlands. PLoS One [Internet]. 2013 [cited 2018 Aug 29];8(12):e81959. Available from: http://www.ncbi.nlm.nih.gov/pubmed/24367496

7. Kramer MS, Berg C, Abenhaim H, Dahhou M, Rouleau J, Mehrabadi A, et al. Incidence, risk factors, and temporal trends in severe postpartum hemorrhage. Am J Obstet Gynecol [Internet]. 2013 Nov [cited 2018 Aug 29];209(5):449.e1-449.e7. Available from: http://www.ncbi.nlm.nih.gov/ pubmed/23871950

8. Breathnach F, Geary M. Uterine Atony: Definition, Prevention, Nonsurgical Management, and Uterine Tamponade. Semin Perinato [Internet]. 2009 Apr [cited 2018 Aug 29];33(2):82-7. Available from: http://www.ncbi.nlm.nih.gov/pubmed/19324236
9. Von Schmidt auf Altenstadt JF, Hukkelhoven CWPM, van Roosmalen J, Bloemenkamp KWM. Pre-eclampsia increases the risk of postpartum haemorrhage: a nationwide cohort study in the Netherlands. PLoS One [Internet]. 2013 [cited 2018 Aug 29];8(12):e81959. Available from: http://www.ncbi.nlm.nih.gov/pubmed/24367496

10. Talledo, L., Talledo, C., \& Pachas P. Incidencia e indicaciones de cesárea practicadas a pacientes ges- tantes de un hospital público de Piura, Perú. 2017;35-8.

11. Rouse DJ, Leindecker S, Landon M, Bloom SL, Varner MW, Moawad $\mathrm{AH}$, et al. The MFMU Cesarean Registry: Uterine atony after primary cesarean delivery. Am J Obstet Gynecol [Internet]. 2005 Sep [cited 2018 Aug 29];193(3):1056-60. Available from: http://www.ncbi.nlm.nih.gov/ pubmed/16157111

12. Say L, Chou D, Gemmill A, Tunçalp Ö, Moller AB, Daniels J, et al. Global causes of maternal death: A WHO systematic analysis. Lancet Glob Heal. 2014;2(6).

13. Alkema L, Chou D, Hogan D, Zhang S, Moller A-B, Gemmill A, et al. Global, regional, and national levels and trends in maternal mortality between 1990 and 2015, with scenario-based projections to 2030: a systematic analysis by the UN Maternal Mortality Estimation Inter-Agency Group. Lancet [Internet]. 2016 Jan 30 [cited 2018 Sep 4];387(10017):462-74. Available from: http://www.ncbi.nlm.nih.gov/ pubmed/26584737

14. Butwick AJ, Ramachandran B, Hegde P, Riley ET, El-Sayed YY, Nelson LM. Risk Factors for Severe Postpartum Hemorrhage After Cesarean Delivery. Anesth Analg [Internet]. 2017 Aug [cited 2018 Aug 29];125(2):523-32. Available from: http://www.ncbi.nlm.nih.gov/ pubmed/28277324

15. Machado C. S. Factores Asociados A La Atonía Uterina En Puérperas Del Hospital Uldarico Rocca Fernandezvilla El Salvador En El Período 
Enero-Diciembre 2014 [Internet].Universidad San Martín de Porres;2017 [cited 2018 Sep 4]. Available from: http://www.repositorioacademico. usmp.edu.pe/bitstream/usmp/2684/1/machado_co

16. Augusto M, Cusirramos G, De JA, Vargas LC. Factores Asociados A Hemorragia Post Parto Inmediato Por Atonia Uterina En El Hospital Guillermo Almenara Irigoyen Durante El Periodo Enero - Septiembre Del 2015 [Internet]. Universidad Ricardo Palma; 2015 [cited 2018 Aug 31]. Available from: http://cybertesis.urp.edu.pe/bitstream/urp/520/1/ Gil_m.pdf

17. Nair M, Choudhury MK, Choudhury SS, Kakoty SD, Sarma UC, Webster $\mathrm{P}$, et al. Association between maternal anaemia and pregnancy outcomes: a cohort study in Assam, India. BMJ Glob Heal [Internet] 2016 [cited 2018 Aug 29];1(1):e000026. Available from: http://www. ncbi.nlm.nih.gov/pubmed/28588921

18. Vendittelli F, Barasinski C, Pereira B, Lémery D, HERA Group the $H$. Incidence of immediate postpartum hemorrhages in French maternity units: a prospective observational study (HERA study). BMC Pregnancy Childbirth [Internet]. 2016 [cited 2018 Aug 29];16(1):242. Available from: http://www.ncbi.nlm.nih.gov/pubmed/27552986

19. Said AS, Manji KP. Risk factors and outcomes of fetal macrosomia in a tertiary centre in Tanzania: a case-control study. BMC Pregnancy Childbirth [Internet]. 2016 [cited 2018 Aug 29];16(1):243. Available from: http://www.ncbi.nlm.nih.gov/pubmed/27557930

20. Butwick AJ, Carvalho B, El-Sayed YY. Risk factors for obstetric morbidity in patients with uterine atony undergoing caesarean delivery. $\mathrm{Br} J$ Anaesth [Internet]. 2014 Oct [cited 2018 Aug 29];113(4):661-8. Available from: http://www.ncbi.nlm.nih.gov/pubmed/24907281
21. Wetta LA, Szychowski JM, Seals S, Mancuso MS, Biggio JR, Tita ATN. Risk factors for uterine atony/postpartum hemorrhage requiring treatment after vaginal delivery. Am J Obstet Gynecol [Internet]. 2013 Jul [cited 2018 Aug 29];209(1):51.e1-6. Available from: http://www.ncbi. nlm.nih.gov/pubmed/23507549

22. Benigno J, Tercero P. Incidencia de la Hemorragia Postparto por Atonia Uterina [Internet]. Universidad de San Carlos de Guatemala; 2014 [cited 2018 Sep 4]. Available from: http://www.repositorio.usac. edu.gt/1662/1/05_9384.pdf

23. Cavazos-Rehg PA, Krauss MJ, Spitznagel EL, Bommarito K, Madden T, Olsen MA, et al. Maternal Age and Risk of Labor and Delivery Complications. Matern Child Health J [Internet]. 2015 Jun 4 [cited 2018 Aug 29];19(6):1202-11. Available from: http://www.ncbi.nlm.nih.gov/ pubmed/25366100

24. Paglia MJ, Grotegut CA, Johnson LNC, Thames B, James AH. Body Mass Index and Severe Postpartum Hemorrhage. Gynecol Obstet Invest [Internet]. 2012 [cited 2018 Aug 29];73(1):70-4. Available from: http:// www.ncbi.nlm.nih.gov/pubmed/21921570

25. Fyfe EM, Thompson JMD, Anderson NH, Groom KM, McCowan LM. Maternal obesity and postpartum haemorrhage after vaginal and caesarean delivery among nulliparous women at term: a retrospective cohort study. BMC Pregnancy Childbirth [Internet]. 2012 Oct 18 [cited 2018 Aug 29];12:112. Available from: http://www.ncbi.nlm.nih.gov/ pubmed/23078042

Consulte la Versión Electrónica de la Revista: Facultad de Medicina Humana Universidad Ricardo Palma http://revistas.urp.edu.pe/index.php/RFMH<smiles>C1=[In]CC1</smiles>

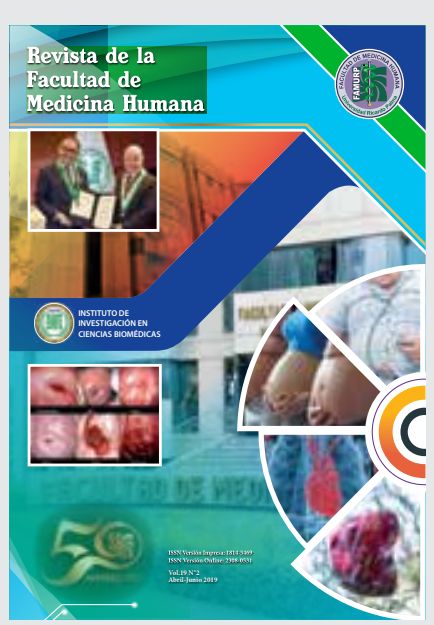

